\title{
ADAPTIVE TOOLPATH FOR 3-AXIS MILLING OF THIN WALLED PARTS
}

\author{
N. Grossi ${ }^{1 *}$, A. Scippa ${ }^{1}$, L. Croppi ${ }^{1}$, L. Morelli ${ }^{1}$, G. Campatelli ${ }^{1}$ \\ ${ }^{1}$ Department of Industrial Engineering, University of Firenze, Via di Santa Marta 3, 50139, Firenze, Italy \\ *Corresponding author; e-mail: niccolo.grossi@unifi.it
}

\begin{abstract}
In this paper a technique to compute the 3-axis toolpath for a thin-wall component is presented aiming at maximizing the engagement conditions, keeping the geometry in tolerance. The toolpath generation is based on the static deflection of the component, predicted by coupling a mechanistic model of the cutting forces with a FE model of the workpiece, including, at each machining step, material removal mechanism. The algorithm follows the milling cycle in the reverse order: starts from the finished part, computes the maximum allowable radial depth of cut, and, adding material accordingly, generates the toolpath until the stock is built. The proposed technique has been experimentally validated, proving its effectiveness.
\end{abstract}

\section{Keywords:}

Toolpath; Milling; Thin-wall workpiece

\section{INTRODUCTION}

The productivity of the milling process of flexible thin-wall parts, typical of the aerospace and energy sectors, is still limited by accuracy issues. Indeed, thin-wall components low rigidity entails errors on the surface caused by static deflection [Sagherian 1990] and vibrations [Budak 2012]. Focusing on dimensional tolerance (i.e., workpiece geometry errors), the key factor impacting on the accuracy is to be found in the workpiece static deflection caused by the cutting forces [Budak 1995]. Since in-process methods to tackle this issue [Kolluru 2013; Huang 2018] are expensive, time-consuming and difficult to be applied, virtual predictive techniques are the preferable choice to build an integrated approach. These methods compute workpiece displacements on the basis of cutting forces and workpiece compliance.

Different strategies have been proposed in literature to both handle the workpiece deflection and ensure the required tolerance: the ones focused on error compensation through a modified toolpath [Ratchev 2005; Rao 2006] and the ones based on the optimization of the process parameters (e.g., radial depth of cut) [Koike 2013; Wang 2017]. Rao and Rao [Rao 2006] developed an error compensation technique, which employs an analytical/numerical model to predict the part deflection that is adopted to compensate the toolpath. Ratchev et al. [Ratchev 2005], instead, starting from an analytical force model integrated with a Finite Element (FE) model, estimate the workpiece deflection, which is then used to offset the toolpath. The main disadvantage of compensation methods in 3-axis milling operations is that tool helix angle produces a variable surface error along the axial depth of cut direction [Desai 2012], hence hardly to be compensated by a simple translation of the toolpath. On the other hand, Wang et al. [Wang 2017] and Koike et al. [Koike 2013] proposed a parameter selection system to deal with workpiece deflections. Essentially, constant axial and radial depths of cut, chosen on maximum allowable deflection basis, are used to build blocks on the finished workpiece. Each one of these blocks represents a volume of the material that should be removed to obtain the final shape of the component. Cutting sequence is defined starting from the finished part adding blocks according to the stiffness of the workpiece, till the whole stock is created. The main disadvantage of the method relies on the cutting parameters estimation that considers the predicted deflection error without compensating it. Moreover, since it is based on constant cutting parameters, depths of cut are constrained by the most flexible point of the component, leading to low productivity, especially in the stiffest areas of the workpiece.

This paper presents a technique, for 3-axis milling operations, that generates an optimized toolpath ensuring the geometric tolerance on a thin-wall component. This strategy combines the benefits of the error compensation methods and parameters selection approaches and it considers the surface error variation along the axial depth of cut. Indeed, the method compensates the mean deflection, which causes the geometric error and selects the process parameters to ensure that the deviation of the deflection, which cannot be compensated, satisfies the tolerance. In detail, the proposed method couples the actual workpiece stiffness during the material removal process, computed using a FE model based on 2D shell elements (Section 2.1), with the error prediction along the axial depth of cut based on the algorithm presented in [Desai 2012] (Section 2.3). The algorithm starts from the final geometry, finding the most suitable point (i.e., end of the toolpath). Then it proceeds to the next points, adding material (according to the computed radial depth of cut), following the milling cycle in the reverse order, as in [Wang 2017], but considering a variable radial depth of cut (Section 2.2). Elaborating the order of the identified points and related radial depth of cut, optimized toolpath is generated (Section 2.4). The proposed technique has been 
experimentally validated on the 3 -axis milling of a blade (Section 3).

\section{PROPOSED APPROACH}

The proposed approach aims at computing the most suitable toolpath for 3-axis milling of thin-wall components to meet the target tolerance. The main features are: i) best cutting sequence identification, ii) radial depth of cut computation and iii) compensation of the surface error on the toolpath. To achieve the goals, the method, schematized in Fig. 1, is composed by several blocks summarized in four steps:

1. Numerical model and workpiece stiffness prediction;

2. Selection of path (cutting sequence);

3. Error along the axial depth of cut;

4. Toolpath generation.

Using the input (process parameters and tool data), the method reconstructs the best toolpath in a reverse order: starting from the finished part till reaching the stock geometry. First, the numerical model of the finished part is created by discretizing its geometry (i.e., FE model). The algorithm starts the loop by selecting the best path (i.e., the order of points). On each point in sequence, the radial depth of cut computation is performed. Radial depth of cut is selected as the highest value that allows to meet the target tolerance (input of the algorithm). Surface error is estimated by a dedicated algorithm that exploits cutting force prediction and static deformation of the workpiece in the cutting point, computed using FE analysis on the model. Once the radial depth of cut is identified, workpiece allowance and numerical model are updated. Radial depth of cut is computed for all the points along the path and the procedure is repeated till the complete stock geometry is obtained. The sequence of paths (i.e., passes), radia depths of cut and surface errors are then used to build the toolpath, starting from the end of the computed cycle to the first step (i.e., finished part). The algorithm, developed in Matlab $^{\circledR}$, automatically arranges the FE analysis, performed using MSC Nastran. In the following sub-sections, the different blocks of the proposed technique are examined in depth.

\subsection{FE model and workpiece stiffness prediction}

Predicting workpiece stiffness is not a straightforward process, since the workpiece changes its geometry and hence its behavior during the machining cycle. Different strategies have been proposed in literature [Budak 2006; Ratchev 2006; Huang 2018], most of them implies the use of Finite Element (FE) models, a convenient way to enable a virtual identification of workpiece behavior at different points and different machining steps. Ratchev et al. [Ratchev 2006] developed an error compensation strategy for single pass peripheral milling based on FE method, while Budak [Budak 2006] studied static displacements of cantilever plates milling with slender end-mills. Other authors simulate thin-wall components behavior for dynamics prediction purpose: Bolsunovskiy et al. [Bolsunovskiy 2013] propose a method to compute the best spindle speeds to reduce the forced vibrations based on FE models of the thin-wall components, Tuyusuz et al. [Tuysuz 2017] applied a reduced modeling technique on a full FE model of a thin-wall structure to predict chatter. All these works are based on 3D solid elements FE models, that for thin-wall components implies the use of small dimension elements, leading to high computational cost. This could be unacceptable when stiffness prediction should be performed several times to study workpiece deflection along the toolpath. In this work, the use of 2D shell elements is proposed with a twofold advantage: i) reducing computational efforts, ii) easing both the automatic generation and updating of the thin-wall structure.

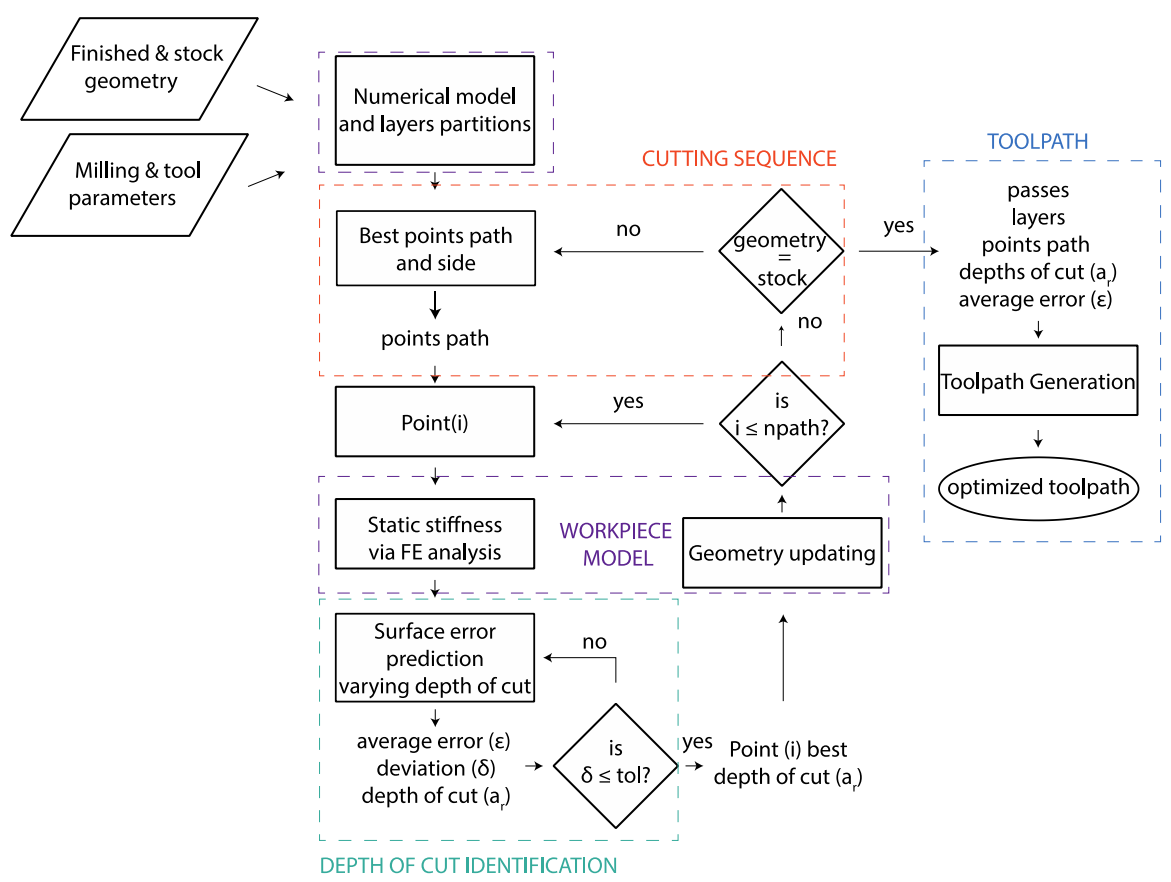

Fig. 1: Proposed approach scheme. 


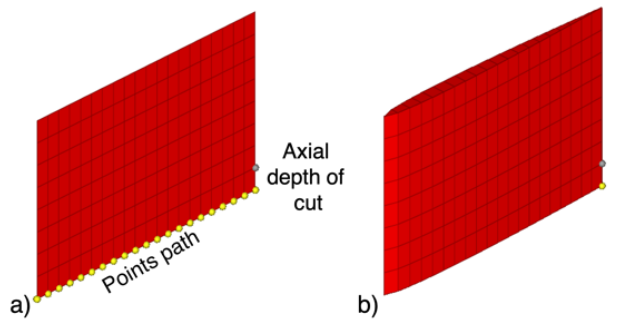

Fig. 2: Shell elements representation in (a) $2 D$ (b) $3 D$.

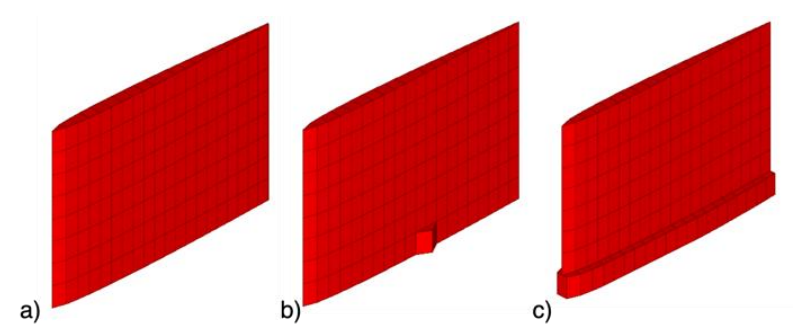

Fig. 3: Thickness updating (a) finished part (b) first point updating (c) full pass updating.

Shell elements are suitable for thin-wall components and ensure the modeling of complex structure by using the midsurface and variable thickness. The nodes on the shell mid line are the ones in which the optimal radial depth of cut is evaluated. On the axial direction two nodes are located limiting the axial depth of cut, as exemplified in Fig. 2.

As previously mentioned, during the algorithm the mesh is automatic updated, allowing a fast generation of the toolpath. This is possible thanks to few operations: i) node id are numbered in a specific order to be used by the algorithm to compile the analysis deck and launch the FE solver (e.g., Nastran) ii) shell elements thickness will be based on the specific thickness values given to the nodes (CQUAD4 card in Nastran [Software Corporation 2010]). This allows the procedure to automatically update the thickness of the nodes at each step by adding the computed radial depth of cut. In Fig. 3 an example of mesh updating procedure is presented. The algorithm starts with the mesh of the finished part (Fig. 3a), at the first point, radial depth of cut is computed and thickness of the part is updated on the shell element (Fig. 3b), this procedure continues till all the nodes are analyzed and mesh updated (Fig. 3c). On the updated FE model of the workpiece at each step, static stiffness on the analyzed points is evaluated by performing a linear static analysis (SOL 101 in Nastran).

\subsection{Selection of Points Path}

The first step of the method is the identification of the cutting sequence. The idea is to build the toolpath, analyzing the cycle in the reverse order. The algorithm starts from the finished part and, at each step, finds the point where workpiece displacement due to the cutting forces is minimum. In that point, material is added (and machining allowance is updated) according to the computed depth of cut, which is the maximum allowable value to meet the tolerance (Section 2.3). The algorithm stops when all the machining allowance is added to the final workpiece shape: i.e., stock geometry is achieved. Finally, the sequence is reversed to obtain the removal process. Cutting sequence in 3-axis milling is composed by the path on X-Y plane (points path) (Fig. 1). The definition of the points path is carried out analyzing all the nodes to be machined on the layer. A static simulation for all the nodes of the layer is performed and the stiffest one is selected as the first node to be machined(i.e., the end of the path). In this work, the method is developed for open thin-wall components that needs to be machined on both sides. Therefore, the points path will pass through the same node twice. A procedure as proposed by Wang et al. [Wang 2017] would require the computation of the workpiece static stiffness in all the machinable points at every step of the machining cycle (i.e., each time material is added (Fig. 3b)), leading to a very high computational cost. On the contrary, since a continuous machining cycle is preferable (as pointed out also in [Wang 2017]), it is only required to define the first point and which side machines first. The side is selected based on the operation type (up or down milling) in order to reach the most flexible node (i.e., lowest static stiffness) on both sides when most of the material is still to be machined. Indeed, material on the part increases the local stiffness of the component, decreasing its deflection. Once the points path is defined, nodes in sequence will be analyzed to compute the maximum radial depth of cut allowable to meet the target tolerance, as explained in the next sub-section.

\subsection{Error Along the Axial Depth of Cut}

In peripheral milling, machined surface is generated at the instant in which tool pass over it (cutting edge perpendicular to the surface). Due to the helical nature of cutter, the axial location of surface generation point changes continuously with cutter rotation and the surface is created at different time instants. Since cutting forces are not constant, tool displacement assumes a certain distribution along the tool axis. Therefore, the surface error caused by the deflection of both the workpiece and the tool will change along the axial depth of cut and cannot be simply compensated by translating the original toolpath.

In this work, surface error along the axial depth of cut is predicted by the formulations presented by Desai and Rao [Desai 2012], extended to workpiece deflection, and a tailored compensation strategy was adopted. The surface error distribution depends on several factors, such as milling strategy (up or down milling), tool geometry (number of flutes and helix angle) and process parameters (axial and radial depth of cut). Starting from these input values, the three characteristic angles can be computed, $\alpha_{\text {en }}$ (radial engagement angle) $\alpha_{s w}$ (axial engagement angle) and $\varphi_{p}$ (pitch angle), and used in the formulations to define the error shape. Error magnitude will depend of cutting forces and tool-workpiece stiffness. In this work, cutting force prediction is based on mechanistic force model: cutting forces are predicted by the following equations:

$$
d F_{t}=K_{t} h d b ; \quad d F_{r}=K_{r} h d b ; \quad d F_{a}=K_{a} h d b
$$

where $F_{t}, F_{r}, F_{a}$ are the tangential, radial and axial components of the cutting force respectively, $\mathrm{h}$ is the uncut chip thickness and $\mathrm{db}$ is the chip width. Each of the force component is described by one coefficient related to material shearing and proportional to the chip thickness $\left(\mathrm{K}_{\mathrm{t}}\right.$, $\mathrm{K}_{\mathrm{r}}, \mathrm{K}_{\mathrm{a}}$ ). As far as stiffness is concerned, tool stiffness will be measured and stored in the algorithm as input, while workpiece stiffness is computed using the FE model presented in the previous section. Using these data surface error distribution can be predicted and used to define toolpath. In the proposed method, two error components are distinguished: an average error $(\varepsilon)$ and a deviation error $(\delta)$, as shown in the example in Fig. 4. The first $(\varepsilon)$ will be compensated by offsetting the original toolpath, while the second $(\delta)$, that cannot be compensated in 3-axis, will be used in the algorithm to compute the most suitable radial depth of cut. Indeed, the value of deviation $(\delta)$ will change with the radial depth of cut and the maximum allowable value will be computed in order to meet the target tolerance. 


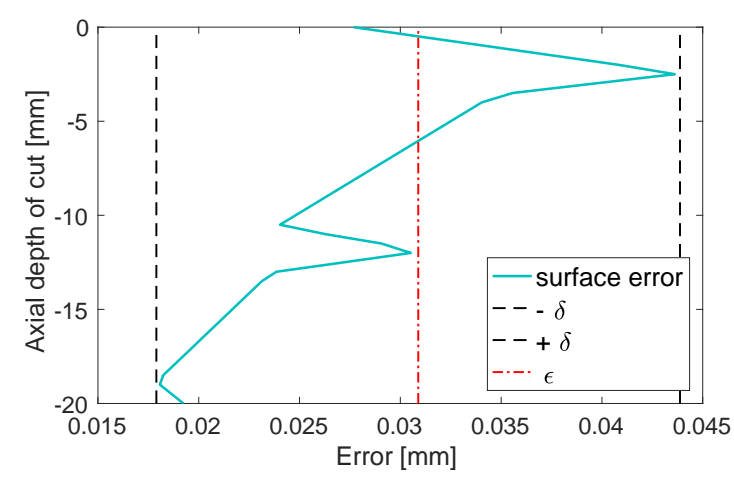

Fig. 4: Example of surface error.

\subsection{Toolpath generation}

The proposed algorithm (described in the previous sections), automatically selects: i) the Z-axis position (i.e., layer), ii) points path and iii) radial depths of cut of the different passes to build the stock, starting from the finished part. After collecting these data, toolpath is generated just reversing the passes and the points path and offsetting the stock geometry of $\Delta$ :

$$
\Delta^{i}=\sum a_{r}^{f}+a_{r}^{i}+\varepsilon
$$

where $a_{r}{ }^{\dagger}$ is the radial depth of cut of the following passes on the same node, $a_{r}{ }^{i}$ is the radial depth of cut computed for the specific pass and point, $\varepsilon$ is the average error of the surface to be compensated. The sign of the offset depends on the side of the workpiece, sign of the error $(\varepsilon)$ depends on the operations (down or up-milling).

\section{EXPERIMENTAL VALIDATION}

The proposed approach has been experimentally validated on a 3-axis milling of a thin walled component, consisting in a single layer operation. A NACA 0005 airfoil profile $(60 \times 20 \mathrm{~mm})$, made of aluminum $(6082-T 4)$ has been machined on a DMG MORI DMU 75 machine tool, using a $12 \mathrm{~mm}$ diameter, four-fluted endmill (Garant 202552), starting from a stock with $6 \mathrm{~mm}$ of thickness and $60 \mathrm{~mm}$ overhang out of the clamp.

To apply the proposed strategy, cutting force coefficients (eq. 1) have been computed by using a mechanistic approach, based on the average cutting forces acquired on a specimen of the same material in slotting at 5 different feed per tooth $(0.05-0.1-0.15-0.2-0.25)$ and 2 depths of cut (0.5-1.0 mm) using a Kitsler 9257A table dynamometer. Experiments were replicated 3 times to improve the reliability, resulting coefficients are reported in Tab. 1.The toolpaths have been used to machine the test case on the $\mathrm{CNC}$ machine using the compensation of the cutter radius (G41), measured using on-machine laser system (BLUM LaserControl Micro Compact NT 87). Surfaces have been acquired using the on-machine probe (RENISHAW PowerProbe 60) (Fig. 6b).

Tool static stiffness has been identified analyzing displacement/force Frequency Response Function, acquired using laser displacement sensor (Keyence LKH085) and impact hammer (PCB 086C03) (Fig. 6a). Target tolerance of the part was set to $\pm 0.02 \mathrm{~mm}$ on a single side (i.e., $\pm 0.04 \mathrm{~mm}$ on the thickness of blade). These data along with tool and cutting parameters are summarized in Tab. 1.
Tab. 1: Tool and cutting parameters.

\begin{tabular}{ccc|cc|c}
\hline \multicolumn{2}{c|}{ Tooling } & \multicolumn{3}{c|}{ Tool static stiffness } & Tolerance \\
\hline $\begin{array}{c}\text { Helix } \\
\text { angle }\end{array}$ & $\begin{array}{c}\text { Diameter } \\
(\mathrm{mm})\end{array}$ & $\mathrm{z}$ & $\begin{array}{c}\mathrm{k}_{\mathrm{tx}} \\
(\mathrm{N} / \mathrm{mm})\end{array}$ & $\begin{array}{c}\mathrm{k}_{\mathrm{ty}} \\
(\mathrm{N} / \mathrm{mm})\end{array}$ & $\delta(\mathrm{mm})$ \\
\hline $45^{\circ}$ & 12 & 4 & 4110 & 5710 & \pm 0.02 \\
\hline \multicolumn{5}{c}{ Cutting parameters } & \multicolumn{2}{c}{ Cutting force } & coefficients \\
\hline $\begin{array}{c}v_{c} \\
(\mathrm{~mm})\end{array}$ & $\begin{array}{c}\mathrm{f}_{z} \\
(\mathrm{~m} / \mathrm{min})\end{array}$ & $\begin{array}{c}\mathrm{Ktc} \\
(\mathrm{mm})\end{array}$ & $\begin{array}{c}\mathrm{Krc} \\
\left(\mathrm{N} / \mathrm{mm}^{2}\right)\end{array}$ & $\left(\mathrm{N} / \mathrm{mm}^{2}\right)$ \\
\hline 20 & 200 & 0.1 & 752.9 & 200.5 \\
\hline
\end{tabular}

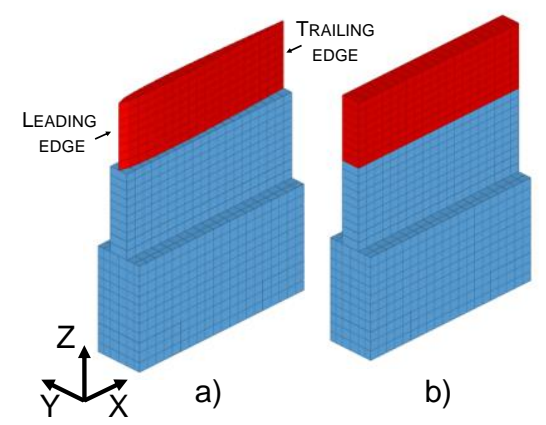

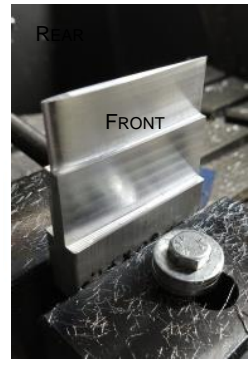

c)

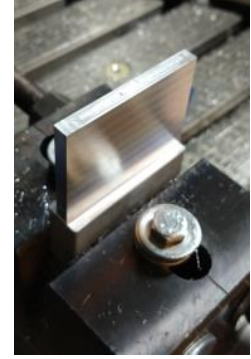

d)
Fig. 5: finished and stock (a)(b) FE models (c)(d) actual. a)



b)

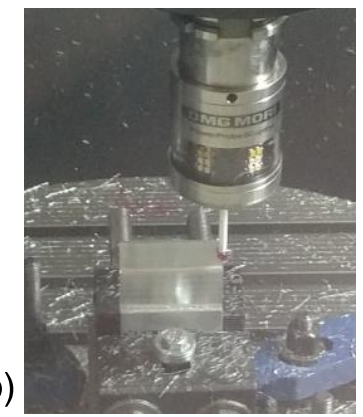

Fig. 6: (a) tool stiffness measure (b) surface acquisition.

\subsection{Toolpath}

Both a traditional toolpath and the optimized toolpath have been tested, using the same cutting parameters. Traditional toolpath consisted in leaving a small machining allowance $(0.5 \mathrm{~mm})$ over the finished part to be removed with the final pass. Finished and stock geometries were input to the developed algorithm and the procedure starts building the FE models (Fig. 5). The FE model of the part is composed by $3203 \times 2.5 \mathrm{~mm}$ shell elements (CQUAD4 in Nastran), while the substrate (part not to be machined) was modelled via solid elements. The following mechanical characteristics were considered for the aluminum: elastic modulus $72.5 \mathrm{GPa}$, density $2680 \mathrm{~kg} / \mathrm{m} 3$, Poisson's ratio 0.34 . Following the steps summarized in the previous sections the method reconstructed the optimized toolpath. 
Depths of cut and errors are computed by FE simulation on the nodes of the model, however generating the part program starting from FEM nodes by linear interpolation will imply a low resolution of the part or a very high computational cost (i.e., if the number of nodes is increased). Therefore, the part program is generated by linear interpolation of the tool on a new denser discretization of the geometry (50 times the number of points per line compared to the nodes of the FE model). Depths of cut and errors for this new discretization geometry are computed by linear interpolation of the values found in the FE model nodes. By doing so, the computation cost is low, while the resolution is high.

Computational efficiency is one of the most important aspect of this kind of approaches. Since several FE analysis must be performed, using full FE models (i.e., large number of degrees of freedom) implies a computational cost difficult-to-handle without the use of dedicated simplification strategies. Altintas et al. [Altintas 2018] proposed a method based on a reduced modeling technique, achieving on a similar test case $\sim 10 \mathrm{~h}$ of computational time on PC with CPU $3.5 \mathrm{GHz}$, decreasing to $\sim 50$ min with a reduction of accuracy of $7 \%$. In this work, to reduce the computational cost, shell elements are adopted along with the aforementioned strategy to build the part program. This led to a computational time of $\sim 17 \mathrm{~min}$ on a similar PC (a laptop CPU $2.4 \mathrm{GHz}$ Intel i5, RAM 4GB) for the whole optimization. Although the comparison is not rigorous since in the first case the approach was focused on only toolpath compensation of ball end-milling of a blade, while in the proposed test case 3 -axis milling is considered and an optimization is performed, it provides an order of magnitude of the computational cost, remarkably low and acceptable for the proposed application.

Traditional and optimized toolpaths are presented in Fig. 7. The two toolpaths differ in both points path and depths of cut. The proposed algorithm (Fig. 7a) selects to entry the cutting from the middle part of the blade, reaching the trailing edge first and then the leading edge. As explained in the previous section, this strategy is based on the stiffness of the component: indeed, the entry is selected on the thickest part, and trailing edge is machined first to keep as maximum material as possible on the blade when machining the most flexible part. a)

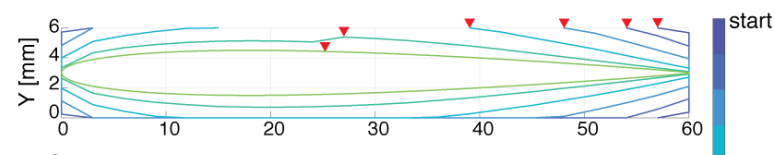

b)
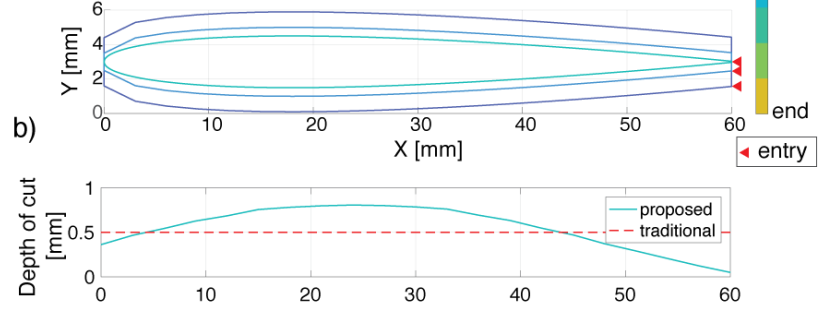

c)

Blade axis position [mm]

Fig. 7: Proposed toolpath (a) traditional toolpath (b) depth of cut of the final pass (c).

On the opposite, a traditional approach that machines the blade starting from the trailing edge (Fig. 7b), ends up in machining the most flexible part at the end of the pass, when all the material is removed.

Moreover, as shown in Fig. 7c, radial depth of cut, constant in the traditional approach, varies in the optimized toolpath: higher depths of cut are selected on the stiffer points and lower ones on the more flexible parts. Due to this selection and the evolution of the machining allowance, the proposed algorithm plans some passes only on specific points (the more compliant), while traditional approach consists in constant depth of cut passes, created by offsetting the airfoil geometry. For this reason, although the number of passes is higher in the proposed toolpath, actual cutting time is only slightly higher: $52 \mathrm{~s}$ against $46 \mathrm{~s}$.

\subsection{Surface results}

Machining surfaces, acquired for both the toolpaths, are used to compute the errors respect to the nominal surfaces. Errors generated on the front side of the blade by the traditional toolpath are presented in Fig. 8, compared to the predicted values. On the front side of the blade, a negative error means a surface thicker than the nominal one, while positive means an overcutting of the surface (opposite on the rear side).
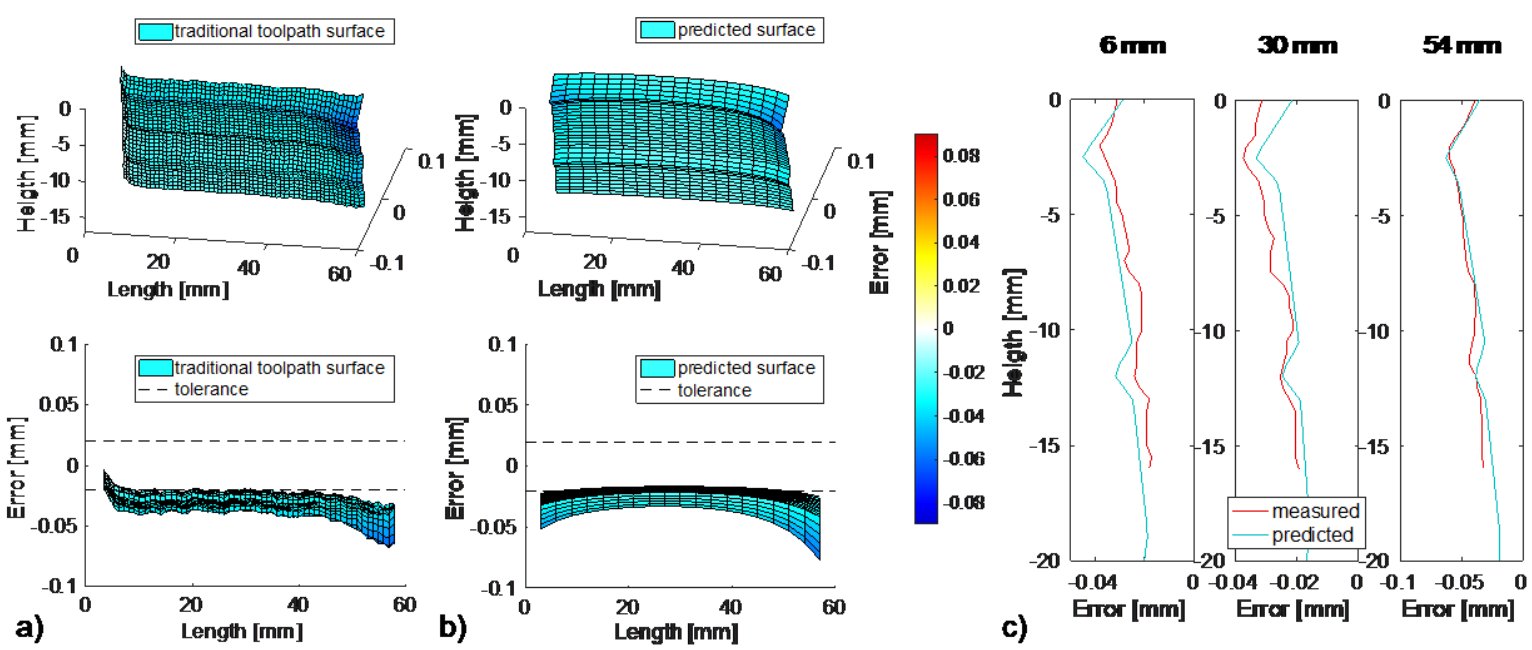

Fig. 8 Machining error on front side of the blade for the traditional toolpath (a) measured (b) predicted (c) sections comparisons. 

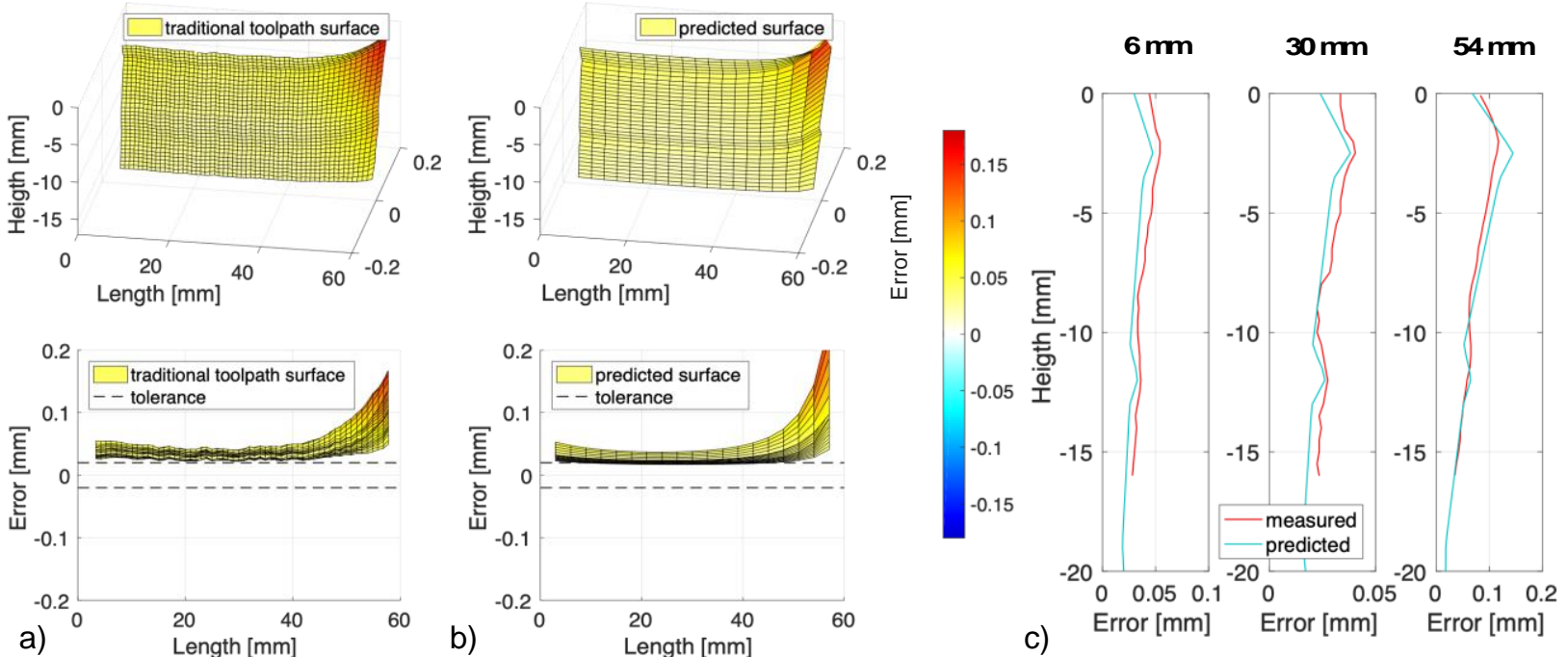

Fig. 9 Machining error on rear side of the blade for the traditional toolpath (a) measured (b) predicted (c) sections comparisons.

For probe size reasons, only $16.5 \mathrm{~mm}$ on the axial direction could be measured. As shown in Fig. 8a, traditional toolpath fails in obtaining a surface in tolerance, returning a thicker blade on the entire surface: error is around $0.04 \mathrm{~mm}$ with peak on the most flexible part (i.e., trailing edge) at around $0.08 \mathrm{~mm}$. This trend was expected, since during downmilling, tool and workpiece static deflection causes an under-cut of the surfaces, not compensated by the traditional approach. Indeed, predicted surface (Fig. 8b) is in line with the experimental results trend, even if an underestimation of the leading edge negative error is found. This discrepancy is probably due to the high curvature of the surface, not accurately modeled by the FE shell model and affecting the direction of application of the cutting forces. Indeed, cutting forces are applied normal to the shell element, in case of the leading edge this approximation is strong, affecting the accuracy of the predictive model. Analyzing three specific sections of the surface error (Fig. $8 \mathrm{c})$, these aspects are highlighted: the traditional approach returns a surface always characterized by a negative error and the proposed model accurately predicts the surfaces both in shape and amplitude.

The same trend is found in the rear side of the blade (Fig. 9a). Surface error (positive in this case, i.e., blade thicker) are out of tolerance with maximum at the trailing edge, as in the other side, but with higher error value (about 0.17 $\mathrm{mm}$ ). This increased error on this side is due to the fact that the tool exits the workpiece at the trailing edge rear side, in this condition workpiece in that point is thinner than on the other side, resulting in high flexibility. As for the front side, the model accurately predicts the surface errors, as shown in Fig. 9b and Fig. 9c.

Optimized toolpath (Fig. 10a) results in a surface with a low error, almost constant all over the geometry, with good match between predicted and experimental values and shape (Fig. 10b). The same discrepancy at the leading edge is found, while at the trailing edge the error is reduced. This is due to the optimized toolpath that considers the flexibility of the system and adapts the radial depth of cut to the specific conditions.
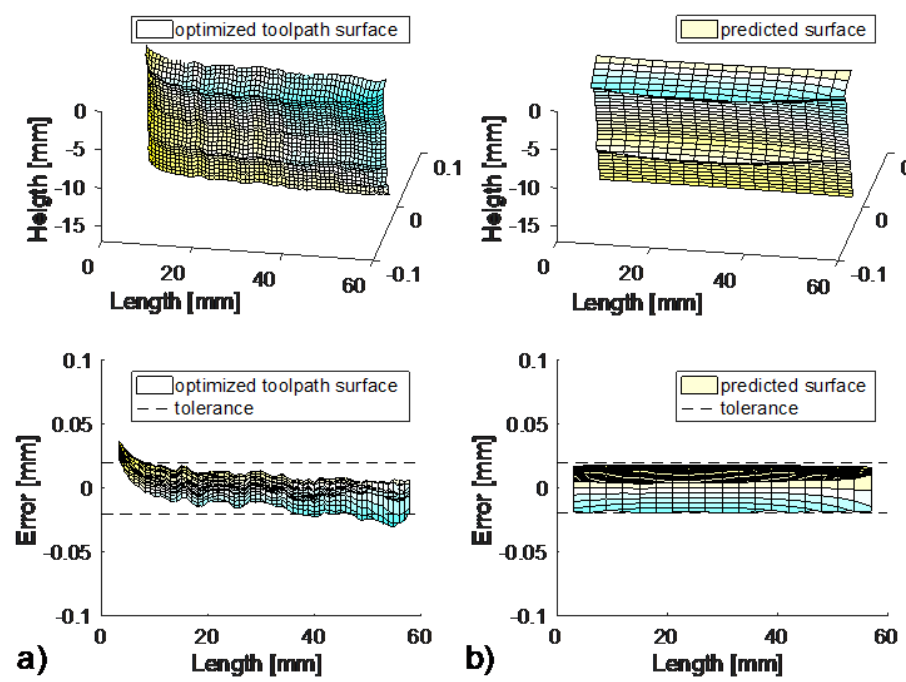

Fig. 10 Machining error on front side of the blade for the optimized toolpath comparisons.

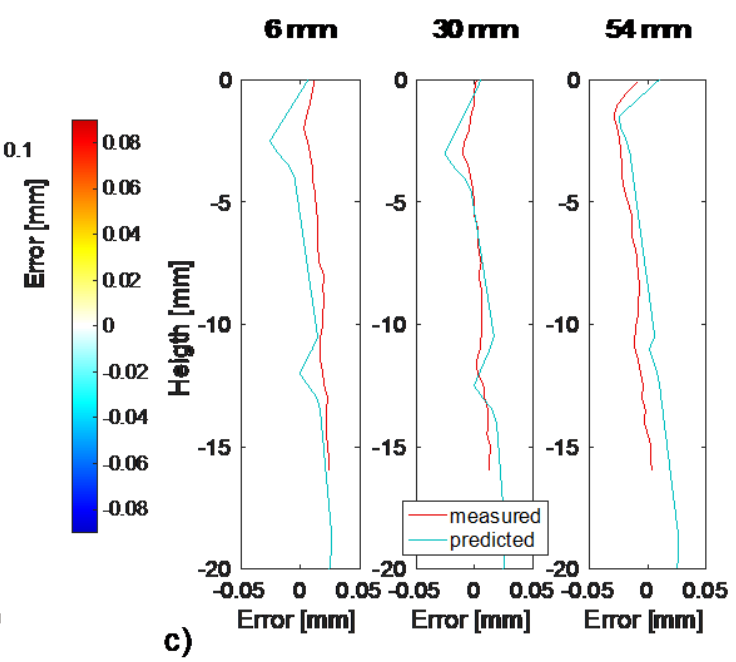

c)



(a) measured (b) predicted (c) sections 

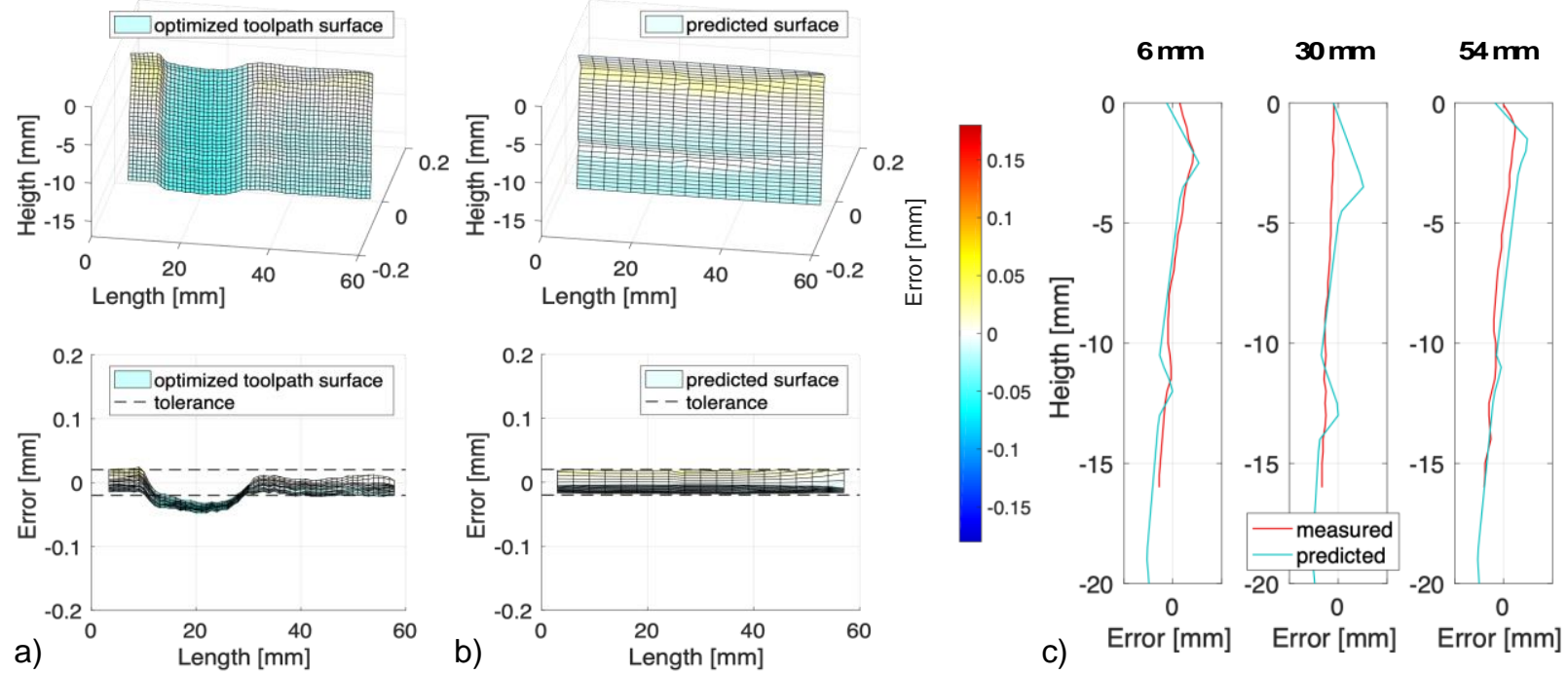

Fig. 11 Machining error on rear side of the blade for the optimized toolpath (a) measured (b) predicted (c) sections comparisons.

As highlighted in Fig. 10c, in contrast with the traditional approach, due to the compensation of the predicted average error $(\varepsilon)$, optimized surface errors are around 0 : surface is thicker at the top and thinner at the bottom. In this way, a symmetric tolerance is obtained $( \pm \delta)$. Obviously, the algorithm could be adapted to ensure any type of tolerance required. The proposed approach manages to keep the error between the target tolerance $( \pm 0.02 \mathrm{~mm})$ with small deviations, while a different toolpath (Fig. 8), that does not consider the flexibility of the system, generates errors out of tolerance. Similar trend is achieved on the rear side (Fig. 11a), with low error and predicted surface in agreement with the experimental one, except for the surface around $20 \mathrm{~mm}$ length where an overcut (negative error) is found. This effect is due to the entry and exit of the tool in the cut. Indeed, to achieve a gradual entry and exit of the tool a radial depth-cut ramp was included, this led to a re-cutting of the part in the entry/exit zone (middle of the blade on the rear side, Fig. 7a). Since this ramp was not included in the proposed algorithm, an overestimation of cutting forces and hence of surface error is predicted, leading to an overcompensation of the mean error $(\varepsilon)$ than required. This "hump" was not found in the traditional toolpath since tool entries and exits the part from the trailing edge. A dedicated strategy should be included in the technique to include this effect and tackle the entry and exit phases.

\section{CONCLUSIONS}

In this paper, a technique to build the toolpath for 3-axis milling operations of thin-walled components is proposed. The method is the first step of the development of an integrated and automatic approach for toolpath generation, aiming at considering the flexibility of both the tool and the workpiece to select cutting parameters (i.e., engagement conditions), overcoming the limits of CAM software, that considers only the kinematic of the process. In this work, this procedure is developed for 3-axis milling: the algorithm generates the optimized toolpath, selecting the best cutting sequence (layers sequence and points path) to reduce the deflection of the component and computes the optimized radial depth of cut to keep the surface error in tolerance, given the other cutting parameters (e.g., axial depth of cut and feed). The algorithm generates the milling cycle in the reverse order: starts from the finished part, computes the maximum allowable radial depth of cut, and adding material accordingly, creates the toolpath until the stock is built

The method was experimentally validated on a thin walled component: a blade NACA 005 made of aluminum. The method was proven to be accurate in predicting surface errors and computing the optimized toolpath, reaching the target tolerance, while a traditional approach fails

Future works will be focused on investigating multi-layers operations and include axial depth of cut and feed to optimize the toolpath, in addition to the radial depth of cut. Furthermore, the presented technique lays the ground for the development of a more general approach, aiming at considering the flexibility of tool/workpiece in the generation of the milling cycle. The method could be extended to simulate both more complex operations, such as 5-axis milling and additional effects, such as forced vibrations and unstable vibrations, since the FE model can be used also to predict workpiece dynamics.

\section{ACKNOWLEDGMENTS}

The authors would like to thank Machine Tool Technology Research Foundation (MTTRF) and its supporters for the loaned machine tool (DMG MORI DMU 75 MonoBlock).

\section{REFERENCES}

[Altintas Tuysuz Habibi and Li 2018] Altintas, Y., Tuysuz, O., et al. Virtual compensation of deflection errors in ball end milling of flexible blades. CIRP Annals, 2018, Vol. 67, No.1, pp. 365-368. doi: 10.1016/j.cirp.2018.03.001.

[Bolsunovskiy Vermel Gubanov Kacharava and Kudryashov 2013] Bolsunovskiy, S., Vermel, V., et al. ThinWalled Part Machining Process Parameters Optimization based on Finite-Element Modeling of Workpiece Vibrations. Procedia CIRP, 2013, Vol. 8, No.0, pp. 276-280. doi: http://dx.doi.org/10.1016/j.procir.2013.06.102.

[Budak 2006] Budak, E. Analytical models for high 
performance milling. Part I: Cutting forces, structural deformations and tolerance integrity. International Journal of Machine Tools and Manufacture, 2006, Vol. 46, No.1213, pp. 1478-1488. doi: 10.1016/J.IJMACHTOOLS.2005.09.009.

[Budak and Altintas 1995] Budak, E. and Altintas, Y. Modeling and avoidance of static form errors in peripheral milling of plates. International Journal of Machine Tools and Manufacture, 1995, Vol. 35, No.3, pp. 459-476. doi: 10.1016/0890-6955(94)P2628-S.

[Budak Tunç Alan and Özgüven 2012] Budak, E., Tunç, L. T., et al. Prediction of workpiece dynamics and its effects on chatter stability in milling. CIRP Annals, 2012, Vol. 61, No.1, pp. 339-342. doi: https://doi.org/10.1016/j.cirp.2012.03.144.

[Desai and Rao 2012] Desai, K. A. and Rao, P. V. M. On cutter deflection surface errors in peripheral milling. Journal of Materials Processing Technology, 2012, Vol. 212, No.11, pp. 2443-2454. doi: 10.1016/j.jmatprotec.2012.07.003.

[Huang Yin Liang Hu and Wu 2018] Huang, N., Yin, C., et al. Error compensation for machining of large thin-walled part with sculptured surface based on on-machine measurement. The International Journal of Advanced Manufacturing Technology, 2018, Vol. 96, No.9, pp. 43454352. doi: 10.1007/s00170-018-1897-x.

[Koike Matsubara and Yamaji 2013] Koike, Y., Matsubara, A., et al. Design method of material removal process for minimizing workpiece displacement at cutting point. CIRP Annals, 2013, Vol. 62, No.1, pp. 419-422. doi: 10.1016/J.CIRP.2013.03.144.

[Kolluru and Axinte 2013] Kolluru, K. and Axinte, D. Coupled interaction of dynamic responses of tool and workpiece in thin wall milling. Journal of Materials Processing Technology, 2013, Vol. 213, No.9, pp. 15651574.

doi: http://dx.doi.org/10.1016/j.jmatprotec.2013.03.018.

[Rao and Rao 2006] Rao, V. S. and Rao, P. V. M. Tool deflection compensation in peripheral milling of curved geometries. International Journal of Machine Tools and Manufacture, 2006, Vol. 46, No.15, pp. 2036-2043. doi: 10.1016/J.IJMACHTOOLS.2006.01.004.

[Ratchev Liu and Becker 2005] Ratchev, S., Liu, S., et al. Error compensation strategy in milling flexible thin-wall parts. Journal of Materials Processing Technology, 2005, Vol. 162-163, , pp. 673-681. doi 10.1016/J.JMATPROTEC.2005.02.192.

[Ratchev Liu Huang and Becker 2006] Ratchev, S., Liu, S., et al. An advanced FEA based force induced error compensation strategy in milling. International Journal of Machine Tools and Manufacture, 2006, Vol. 46, No.5, pp. 542-551. http://dx.doi.org/10.1016/j.jmachtools.2005.06.003.

[Sagherian and Elbestawi 1990] Sagherian, R. and Elbestawi, M. A. A simulation system for improving machining accuracy in milling. Computers in Industry, 1990, Vol. 14, No.4, pp. 293-305. doi: https://doi.org/10.1016/0166-3615(90)90037-P.
[Software Corporation 2010] Software Corporation, M. S. C. MD/MSC Nastran 2010 Quick Reference Guide 2010.

[Tuysuz and Altintas 2017] Tuysuz, O. and Altintas, Y. Frequency Domain Updating of Thin-Walled Workpiece Dynamics Using Reduced Order Substructuring Method in Machining. Journal of Manufacturing Science and Engineering, 2017, Vol. 139, No.7, pp. 71013-71016. Available at: http://dx.doi.org/10.1115/1.4036124.

[Wang Ibaraki and Matsubara 2017] Wang, J., Ibaraki, S., et al. A cutting sequence optimization algorithm to reduce the workpiece deformation in thin-wall machining. Precision Engineering, 2017, Vol. 50, , pp. 506-514. doi: 10.1016/J.PRECISIONENG.2017.07.006. 